Gangi, Lisa

\section{Mammary gland involution studies with cDNA microarrays}

\author{
Lisa Gangi ${ }^{1}$, Garrison 0 wens ${ }^{1}$, Robin Humphreys ${ }^{2}$, Lothar \\ Hennighausen ${ }^{2} \&$ Edison Liu ${ }^{1}$
}

${ }^{1} \mathrm{NCl}$ and ${ }^{2} \mathrm{NIDDK}, \mathrm{NIH}$, Bethesda, M aryland, USA

Mammary gland involution follows the cessation of lactation. Similar to lactogenesis, the involution process requires an ordered sequence of events including the termination of milk production, increased secretion of lactoferrin, apoptosis of the mammary epithelium and remodelling of the extracellular matrix of the gland. A completed cycle of involution requires apoptosis of the epithelial mammary structures involved in milk production, resulting in the loss of the milk-secreting alveoli as the gland returns to a pre-pregnant state. The complex cellular remodelling and cell-specific targeted apoptosis make mammary involution an excellent model system to apply to microarray platforms. The development of a $3 \mathrm{~K}$ mammaryenriched mouse cDNA microarray has enabled us to perform high-throughput hybridization analysis to establish the gene expression profiles associated with the distinct cellular stages of the involution process. We find that a fall in anti-apoptosis genes appears to initiate the process, followed by induction of stressresponse genes. In addition to uncovering many genes involved in cell-cycle regulation, apoptosis, DNA repair and cytoskeletal remodelling, these microarrays have proven to be a powerful tool for gene discovery in epithelial involution.

Gant, Timothy W.

\section{Multiple analysis of gene expression contributing to differential xenobiotic sensitivity}

\author{
T.W. Gant \\ M olecular Toxicology, M edical Research Council, Leicester, UK
}

Some inherited pathological disorders are caused by a single mutation and others have susceptibility components which are heritable, but polygenic (arising from the contribution of several genes) in origin. Often, these genes have biological functions that are not obviously related to the symptoms, and pathology of the disorder, and their contribution is not thus recognized. The same is true when the susceptibility (or resistance) of individuals is assessed in relation to chemically induced toxicity, or in relation to the activity of molecules with pharmacological properties. Working on the premise that any genes which contribute to a susceptibility, or resistance, phenotype may be differentially expressed in relation to those in a normally responsive individual or tissue, we are using microarray technology to screen for differential gene expressions that may be contributing to the phenotype. We have built an arrayer based on the Stanford design, with some modifications, and are using this to produce arrays of up to 2-56K clones (depending on requirement). We are arraying both known genes and cDNA library clones from the rat, mouse and human. Initial analytical work has been carried out on human drug resistance cell lines, and increased expression of some well-characterized genes have been observed (MDR1, GST), as well as some changes of expression which are consistent with other phenotypic traits of these cells. This work is currently being extended, and all relevant findings will be presented.
Ganter, Brigitte

\section{Discovery of septicaemia-related genes using CDNA microarrays}

\author{
Brigitte Ganter \& Jeffrey Libby
}

diaDexus LLC, 3303 O ctavius Drive, Santa Clara, California 95054, USA

Infection induced by gram-negative and gram-positive bacteria results in an enormous host immune response that has two possible outcomes. One in which the organisms are eliminated and the host returns to normal, and the other in which the battle between host and invader leads to disregulation of innate immunity and the development of septic shock. In inflammatory diseases such as septic shock, gene expression patterns of diverse cell types contribute to the pathology. We are currently in the process of monitoring gene expression during different states of infection using cDNA microarrays. mRNA from cultured human monocytic THP1 cells and isolated peripheral blood leukocytes stimulated with the endotoxin LPS or gram-negative bacteria are analysed for this study.

Preliminary microarray data support the finding that inflammation is associated with the expression of many different cytokines, such as interleukin-1, TNF, interleukin- 8 and an abundance of chemokines. Current efforts aim to identify signature expression patterns associated with different states of infection.

Gerhold, David

\section{A gene expression microarray database for drug metabolism and toxicology}

\author{
David Gerhold, Jian Xu, Meiqing Lu, C. Thomas Caskey \\ \& Tom Rushmore
}

M erck Research Labs, West Point, Pennsylvania 19486, USA

The liver is a primary site for metabolism of xenobiotic compounds by enzymes which are dynamically regulated. Gene regulation in the liver also reflects the overall metabolic, energetic and hormonal state of the organism. Thus, we have generated a DNA Chip database to profile drug metabolism and toxic responses in rat liver. Rats were treated with four compounds which induce characteristic cytochrome P-450 genes. Gene expression was assayed in livers of these rats using Affymetrix DNA Chips. Induction of the expected cytochrome P-450 genes was observed, validating the approach. Additional regulatory responses were identified for genes encoding drug-conjugating enzymes and drug transporters for these well-studied compounds. Sex-specific expression of drug metabolic genes was also identified by comparing male and female rat livers. We encountered difficulties with DNA microarray technologies due to heterologous hybridisation between genes which share homology, or simple sequence repeats. We used cytochrome P-450 genes from four subfamilies to evaluate gene-to-gene hybridisation specificity using Affymetrix arrays. Gene-specific hybridisation was maximised by rational selection of DNA sequences to the array on the DNA chip. 